\title{
The spatial location of laser-driven, forward-propagating waves in a National-Ignition-Facility-relevant plasma
}

\author{
J. A. Cobble, J. C. Fernández, N. A. Kurnit, D. S. Montgomery, and R. P. Johnson \\ Los Alamos National Laboratory, Los Alamos, New Mexico 87545 \\ N. Renard-Le Galloudec ${ }^{\text {a) }}$ \\ Laboratoire pour l'Utilisation des Lasers Intenses (LULI) Unité Mixte No. 7605 CNRS, CEA, \\ Ecole Polytechnique, Université Pierre et Marie Curie \\ M. R. Lopez \\ University of Michigan, Ann Arbor, Michigan
}

(Received 14 June 1999; accepted 5 October 1999)

\begin{abstract}
Ion acoustic and electron plasma waves, associated with backward-propagating stimulated Brillouin scattering and stimulated Raman scattering, have been diagnosed in a long-scale-length, nearly homogenous plasma with transverse flow. Thomson scattered light from a probe beam is employed to show that these waves are well localized in space and for a time much shorter than the laser pulse duration. These plasma conditions are relevant to hohlraum design for the National Ignition Facility inertial confinement fusion laser system. [R. Sawicki et al., Fusion Technol. 34, 1097 (1998)].

(C) 2000 American Institute of Physics. [S1070-664X(00)03001-9]
\end{abstract}

\section{INTRODUCTION}

Among the primary obstacles to thermonuclear ignition at the National Ignition Facility (NIF) ${ }^{1}$ are laser-plasma instabilities. The most dangerous of these are stimulated Brillouin scattering (SBS) and stimulated Raman scattering (SRS), particularly convective spatial amplification along the backscattered direction. ${ }^{2}$ For both processes, laser light typically gives up energy to electrostatic waves and scattered light in the plasma. SBS involves laser scattering from ion acoustic waves while SRS is produced together with electron plasma waves, which also lead to superthermal electrons and the threat of fusion capsule preheat.

In spite of much study, ${ }^{3-7}$ the goal of instability control has not been achieved. For example, in ignition-relevant plasmas, the calculated spatial gain for SBS can be enormous, which raises the question about what the saturation mechanism might be. Possibilities include but are not limited to a smaller spatial gain rate than calculated, a smaller gain length than anticipated (perhaps limited by hydrodynamic fluctuations ${ }^{8}$ ), or competition ${ }^{9}$ between instability processes. The purpose of this paper is to address these possibilities by imaging the location of laser-plasma instabilities in a NIFrelevant plasma. On NIF, the plasma is produced and confined in a hohlraum, a laser-driven radiation case used to implode the fusion capsule. ${ }^{10}$ Such a plasma is characterized by low electron density (a few percent $n / n_{\text {crit }}$, where $n_{\text {crit }}$ is the density beyond which a laser beam is totally reflected) and plasma flow away from the hohlraum walls and out of the laser entrance holes. The critical density is given by $n_{\text {crit }}=\pi m c^{2} / e^{2} \lambda^{2}$, where the usual definitions apply for electron mass and charge, the speed of light, and the wavelength of the laser drive. The depth of laser beam penetration

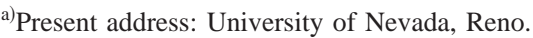

into NIF hohlraum plasmas will be a few millimeters. The experiments reported in this paper mimic conditions near a NIF laser entrance hole (LEH), where a high-intensity laser beam interacts with a low-density plasma having supersonic transverse flow. To be specific, in the NIF LEH, according to Fig. 10.2 of Ref. 10, the plasma is a nearly constant $\sim 5 \%$ $n / n_{\text {crit }}$ for a distance of $1-2 \mathrm{~mm}$ along the beam paths. The flow, roughly down the density gradient, is approximately perpendicular to the direction of the laser beams and is Mach 1 to Mach 2. The electron temperature $T_{e}$ is expected to be $5-6 \mathrm{keV}$. The experimental conditions for the research presented in this paper are similar to NIF in that the plasma density, scale length, flow direction, and flow magnitude are similar to those in the LEH region of a NIF hohlraum. Additionally, the growth rates for SBS and SRS are sufficiently large in our experiment to be in the nonlinear regime, and the waves are strongly damped.

The present experiment is relevant for understanding the interaction near the NIF LEH, except that $T_{e}$ is about a tenth of the NIF temperature. The major effect of this is that while NIF resides in the ponderomotive regime for laser plasma instabilities, the current experiment is in the thermal regime. Much more laser energy than is available on our current laser is needed to better match both $T_{e}$ and plasma size on NIF.

\section{EXPERIMENTAL SETUP}

We performed this experiment at the Trident laser facility ${ }^{11}$ in a surrogate plasma geometry employing three Trident beams. All three laser pulses have a square temporal profile with a duration of $1.2 \mathrm{nsec}$. First, a plasma formation beam creates a plasma of length $\sim 1 \mathrm{~mm}$ and width $\sim 150$ $\mu \mathrm{m}$ by hitting a $1 \mathrm{~mm}$ diameter, $6.7 \mu \mathrm{m}$ thick $\mathrm{CH}$ disk. The geometry but not the time history is shown in Fig. 1(a). The $10^{14} \mathrm{~W} / \mathrm{cm}^{2}, 527 \mathrm{~nm}$ plasma formation beam is line focused 
(a)

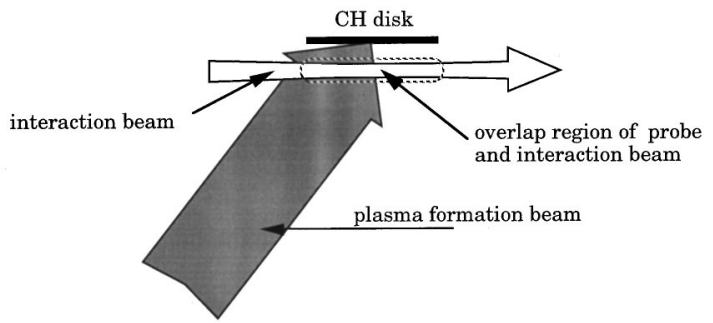

(b)

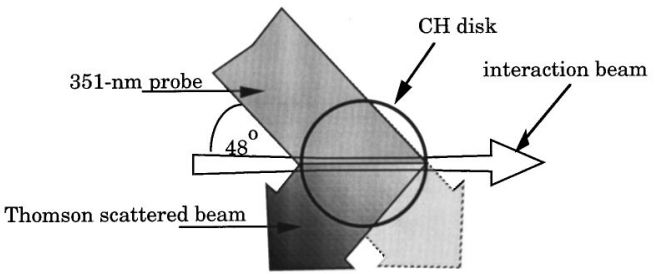

(c)

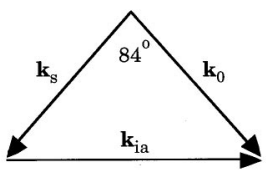

FIG. 1. Geometry of the target and beams: (a) Top view of disk target. (b) Front view. (c) Vector diagram of $351 \mathrm{~nm}$ Thomson scattering from a forward propagating ion acoustic wave created by $527 \mathrm{~nm}$ light from the interaction beam

by passing it through two random phase plates (RPPs). ${ }^{12,13}$ One RPP has a $6 \mathrm{~mm}$ hexagonal pattern; the second RPP pattern is $6 \times 0.8 \mathrm{~mm}$. The angle of incidence of the plasma formation beam is $\sim 40^{\circ}$ in this experiment. The resulting well-characterized plasma ${ }^{14,15}$ falls off exponentially in density away from the disk and is nearly isothermal with an electron temperature of $\sim 500 \mathrm{eV}$ out to at least $500 \mu \mathrm{m}$ from the target plane. After a delay of 1.65 nsec to ensure no interference from effects of the formation beam, a $527 \mathrm{~nm}$ interaction beam is injected at a spacing of $250 \mu \mathrm{m}$ from the face of the disk along the length of the plasma. Its average peak intensity is $\sim 1.2 \times 10^{15} \mathrm{~W} / \mathrm{cm}^{2}$. At the $250 \mu \mathrm{m}$ spacing, the plasma density is $\sim 2 \times 10^{20} \mathrm{~cm}^{-3}\left(\sim 5 \% n_{\text {crit }}\right)$. The focusing lens is $f / 6$, and an RPP with a $6 \mathrm{~mm}$ hexagonal pattern is employed to give a focal spot $150 \mu \mathrm{m}$ in diameter. The measured supersonic plasma flow (Mach number $\sim 2.2)^{15}$ is transverse to the interaction beam which we use to generate SBS and SRS. The density and flow are analogous to the conditions a NIF beam will sample as it enters the hohlraum LEH. Figure 2 shows the anticipated plasma density and temperature for this set up as measured in Ref. 15. We note that because the $\mathrm{CH}$ foil does not burn through, the density holds steady in time and exhibits an exponential spatial profile even after the plasma formation beam is turned off. And, as expected, the isothermal $T_{e}$ profile will decrease from its peak value, measured by Thomson scattering from the plasma formation beam. Also at $1.65 \mathrm{nsec}$ delay, the frequency-tripled third Trident beam is used as a Thomson scattering probe at $351 \mathrm{~nm}$. The probe is line focused by means of a cylindrical lens placed in front of an $f / 11$ focus- (a)

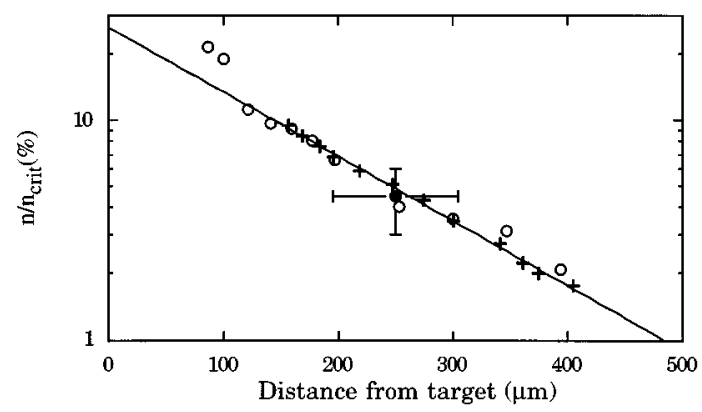

(b)

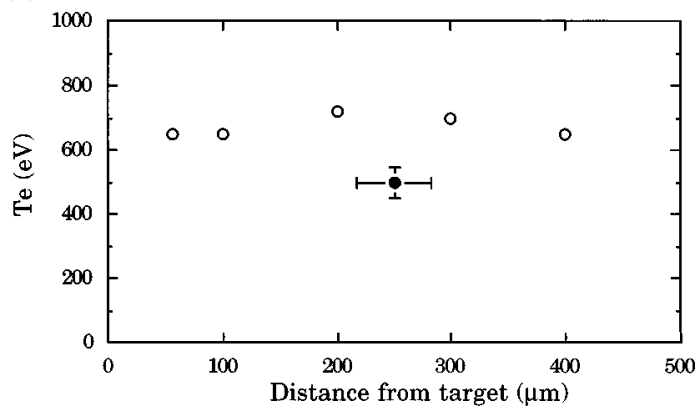

FIG. 2. (a) Plasma density away from the target surface: open circles derive from the magnitude of previous Thomson scattering measurements and crosses are from previous SRS measurements in Ref. 15; the dark circle is from the present experiment. The exponential fit is to published SRS data with an e-folding distance of $\sim 150 \mu \mathrm{m}$. The $\mathrm{x}$ error bar is half the probe radius of $110 \mu \mathrm{m}$. (b) Thomson scattering data showing an isothermal electron temperature away from the target surface: open circles are from Ref. 15; the dark circle shows the present measurement $1.45 \mathrm{nsec}$ after the plasma formation beam is extinguished.

ing lens and runs parallel to the face of the disk, overlapping the interaction beam as indicated in Figs. 1(a) and 1(b). The projected cross section of the probe beam on the plasma is $\geqslant 1 \mathrm{~mm}$ along the interaction beam path and $\sim 220 \mu \mathrm{m}$ wide in the transverse direction. Note that in this open geometry, the plasma density and scale length along the interaction beam direction are comparable to anticipated NIF plasma conditions. The chosen target geometry is the simplest one that allows us to have a low-density, nearly homogeneous plasma on the order of $1 \mathrm{~mm}$ long and, at the same time, a flow transverse to the interaction beam.

The momentum equation for SBS and SRS in a plasma is

$$
\mathbf{k}_{0}=\mathbf{k}_{s}+\mathbf{k}_{\text {ialep }},
$$

where $\mathbf{k}_{0}$ is the interaction beam wave vector, $\mathbf{k}_{s}$ is the scattered light wave vector, and $\mathbf{k}_{\text {ialep }}$ is the wave vector of the ion acoustic or electron plasma wave. For example, for backscattered SBS, $\mathbf{k}_{s} \sim-\mathbf{k}_{0}$, and $\mathbf{k}_{i a} \sim 2 \mathbf{k}_{0}$.

Laser Thomson scattering ${ }^{16}$ is a well-known tool for plasma diagnostics and is used in the collective scattering regime $^{17}$ to detect electrostatic waves driven by instabilities in laser plasmas. Similar to the above equation, the momentum equation for Thomson scattering is

$$
\mathbf{k}_{0}=\mathbf{k}_{s}+\mathbf{k},
$$




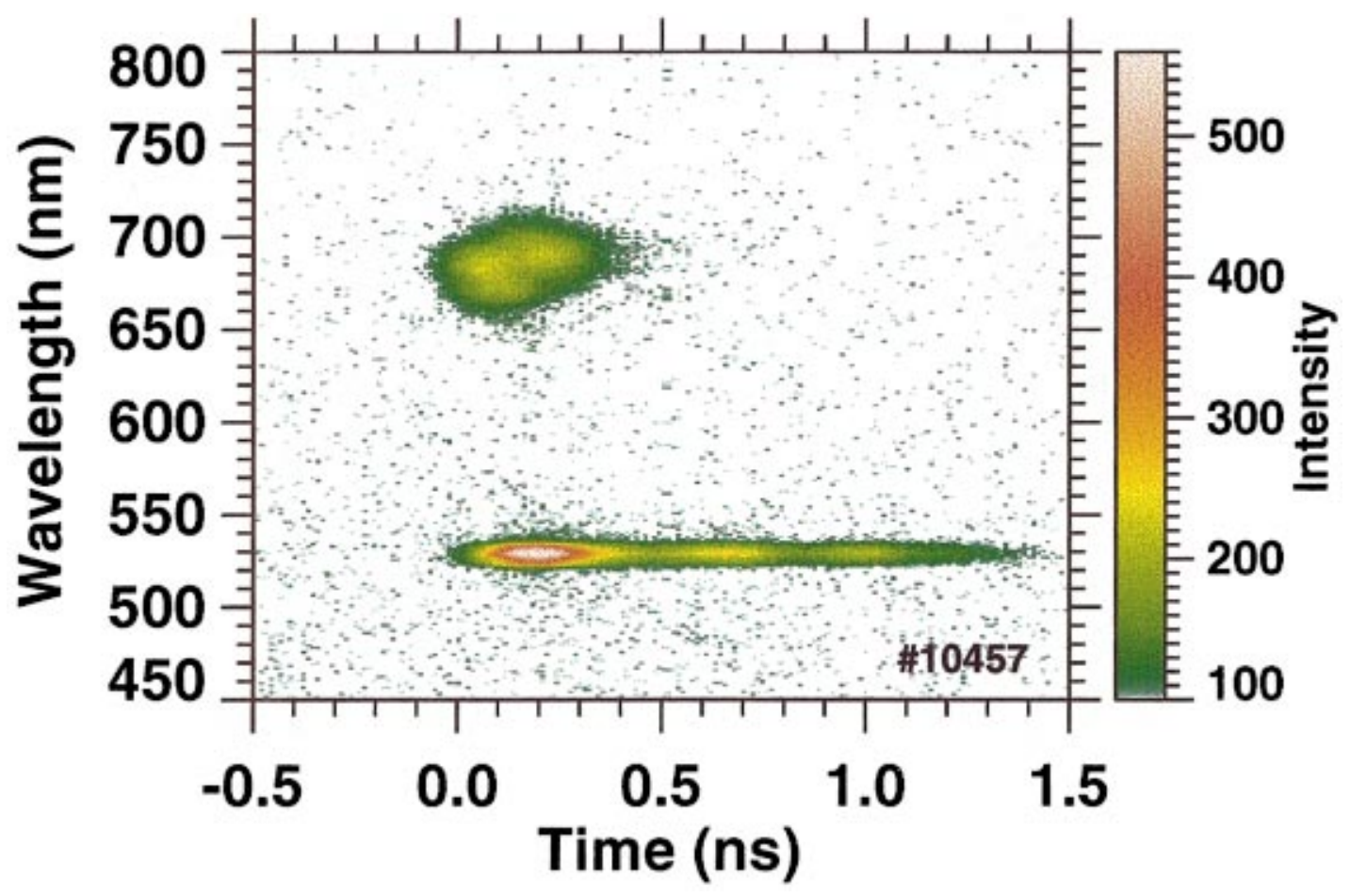

FIG. 3. (Color) Time-resolved spectrum of the backscattered light. The nominal time for the start of the interaction beam is $t=0$.

where $\mathbf{k}_{0}$ is now the wave vector for the probe beam, $\mathbf{k}_{s}$ is the wave vector for the Thomson scattered light, and $\mathbf{k}$ is the Thomson scattering vector. Using Thomson scattering in this experiment, we image the location of electrostatic waves in the plasma associated with SBS or SRS backscatter from the interaction beam. For example, if a $351 \mathrm{~nm}$ Thomson probe beam is set to make an angle of $48^{\circ}$ with the ion acoustic wave $\mathbf{k}_{i a}$ responsible for SBS, then $\mathbf{k}=\mathbf{k}_{i a}$ as depicted in Fig. 1(c). We interpret the strength of the Thomson scattering signal as being indicative of enhanced density fluctuations characteristic of electrostatic wave activity.

Previous similar work includes that of Labaune et al. and Renard et al. who probed exploded foil targets of similar density with a $351 \mathrm{~nm}$ Thomson scattering beam to spatially resolve ion acoustic waves ${ }^{18}$ and electron plasma waves. ${ }^{19}$ Some differences between their studies and the present experiment are that their plasma was not flowing transverse to the interaction beam direction and that in their case, the density profile was parabolic with a relatively short scale length and not flat-topped along the direction of propagation as in the present experiment.

In a hohlraum environment, given the constraints of the scattering angle, Thomson scattering requires a diagnostic window, ${ }^{20}$ which for long-scale-length imaging would compromise the integrity of the hohlraum. The open-geometry Trident configuration with its ease of access provides a welldiagnosed, NIF-relevant, long-scale-length, nearly homogeneous plasma, albeit at a lower electron temperature.

The Thomson scattered light from the $351 \mathrm{~nm}$ probe beam is collected with an $f / 4$ achromatic lens. This enables us to collect broadband spectral signals whether the scattering vector is associated with ion-acoustic or electron plasma waves associated with stimulated backscatter. ( $\mathbf{k}_{s}$ is not the same for both but still within the $7^{\circ}$ half angle of the collection optics.) The scattered light is relayed, divided, and imaged at $10 \times$ magnification (i) on the slit of a filtered streak camera for spatial resolution $(\sim 50 \mu \mathrm{m})$ of either ion acoustic or electron plasma wave activity in the $1 \mathrm{~mm}$ plasma and (ii) into a spectrograph for spectral resolution. The imaging spectrometer output is streaked to acquire a Thomson spectrum from electrostatic waves at a single location in the plasma. A second streaked spectrograph records the time history of the backscattered interaction beam-both the SBS at $527 \mathrm{~nm}$ and the SRS, from which plasma density may be estimated. A portion of the backscatter signal is split off, filtered through a $527 \mathrm{~nm}$ bandpass filter, and used for a measurement of absolute SBS backscatter with a vacuum photodiode, which has greater dynamic range than the streak camera. The same beam splitter which directs backscatter toward this pair of diagnostics reflects incident laser light to an uncoated, wedged glass aligned to retroreflect incident laser light back through the beam splitter into the same detectors. Because the beam splitter reflectivity is common to both the backscattered SBS and the reflected incident beam path, the detectors are self- calibrated automatically for SBS. Finally, a diffusing, annular $\mathrm{Al}$ plate around the interaction beam cone is used to detect backscattered light just outside of the lens cone. Depending on the filtration, this diagnostic, called a near backscatter imager (NBI), ${ }^{21}$ images either SBS or SRS light from the diffusing plate with a CCD camera. The NBI has been calibrated for SBS with a $527 \mathrm{~nm}$ laser beam.

To check the reproducibility of this experiment with regard to previous experimental campaigns, we monitored plasma density and temperature with scattered light signals. Figure 3 shows a streaked backscattered spectrum from the 

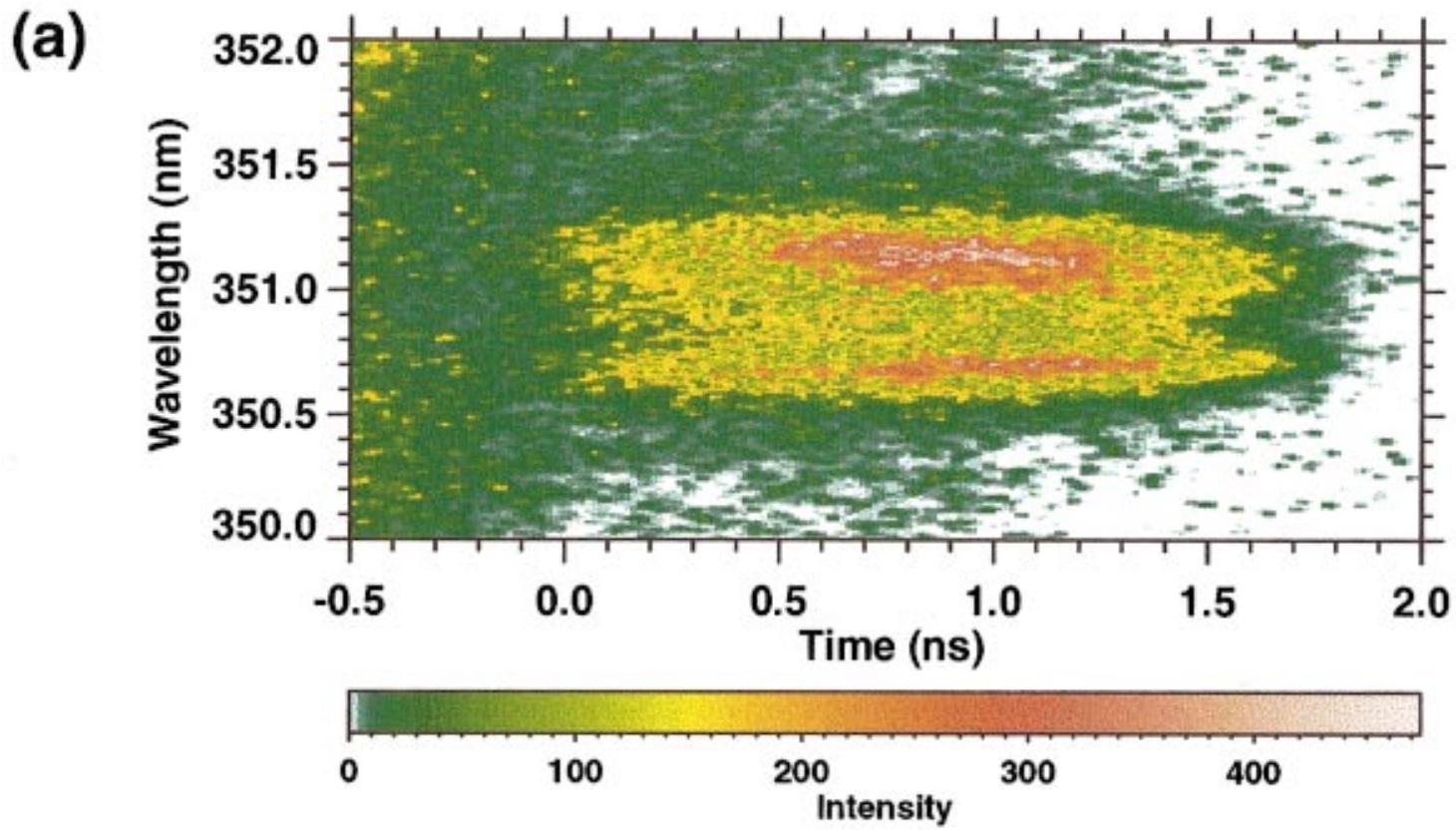

(b)

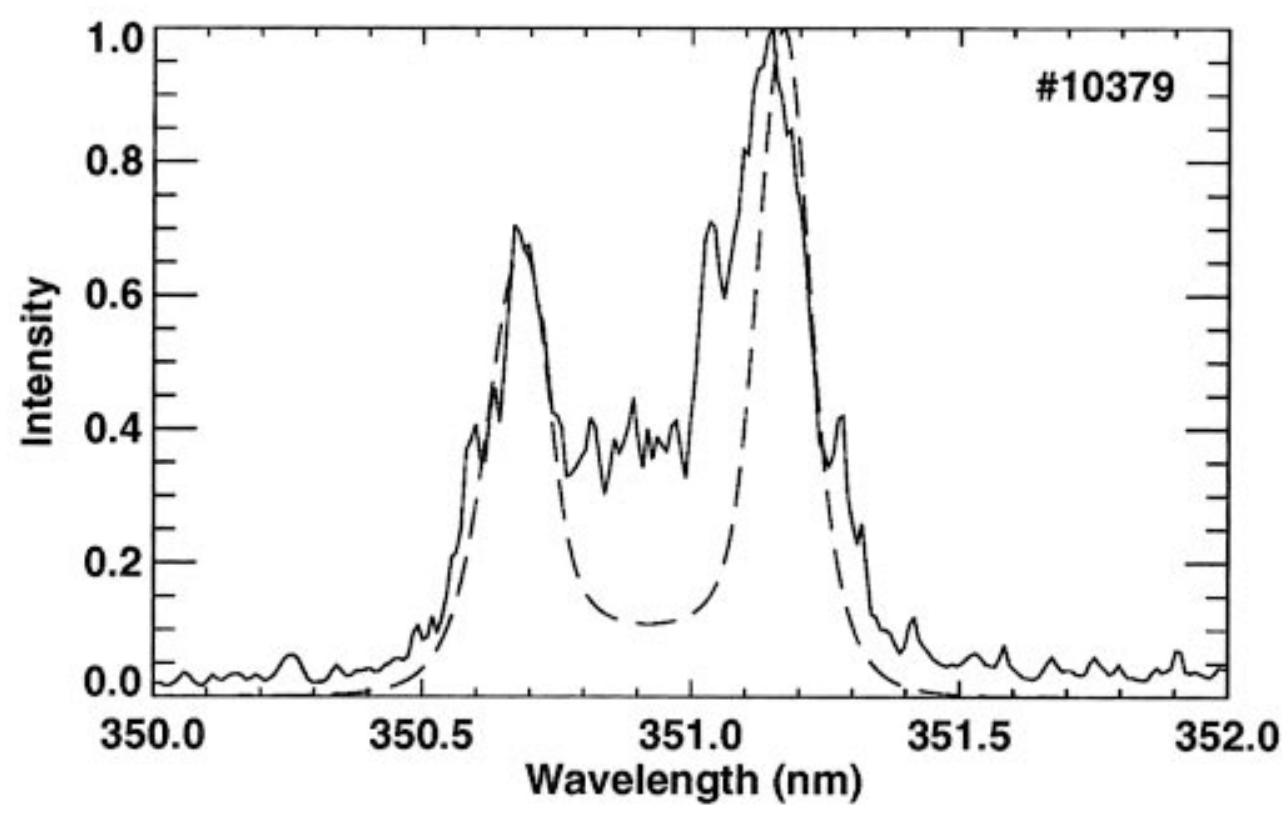

FIG. 4. (Color) (a) Time-resolved Thomson spectrum from the ion acoustic waves associated with stimulated Brillouin backscatter from the interaction beam. (b) A lineout at $1 \mathrm{nsec}$ with the theoretical profile indicated by dashes.

interaction beam. The signal at $680-700 \mathrm{~nm}$ is SRS, from which we derive the plasma density accounting for the small Bohm-Gross correction for plasma temperature. ${ }^{22}$ For this series of experiments, the electron density range is $1.2-2.4$ $\times 10^{20} \mathrm{~cm}^{-3}\left(3 \%-6 \% n_{\text {crit }}\right)$. This inferred density from the SRS backscatter is indicated in Fig. 2(a) as the dark circle with error bars. It is seen to agree with our expectations, i.e., our previous profile from Ref. 15 . We note that the variation in density, the error bar, is consistent with the plasma profile across the width of overlap of the interaction beam and Thomson probe beam. Thomson scattering spectra from undriven ion acoustic waves are used to measure the plasma temperature, ${ }^{16}$ typically in the range $T_{e} \sim 500 \pm 50 \mathrm{eV}$ and
$T_{i} \sim 150 \pm 50 \mathrm{eV}$. Figure 4(a) shows a streaked Thomson spectrum form thermal-level ion acoustic waves. The profile in Fig. 4(b) is taken at 1 nsec after the start of the probe beam- $1.45 \mathrm{nsec}$ after the plasma formation beam is turned off. The $T_{e}$ obtained from this measurement is plotted in Fig. 2(b) and shows a modest electron cooling from peak values obtained while the heater is on. The blue shift in Fig. 4(b) of the whole spectrum from $351.0 \mathrm{~nm}$ indicates a small flow component opposite to the direction of $\mathbf{k}_{i a}$. This is consistent with a moderately diverging plasma near the input edge of the plasma. The theoretical fit is better on the blue side of the spectrum. On the red side, the bump at $351.0 \mathrm{~nm}$ could 
(a)
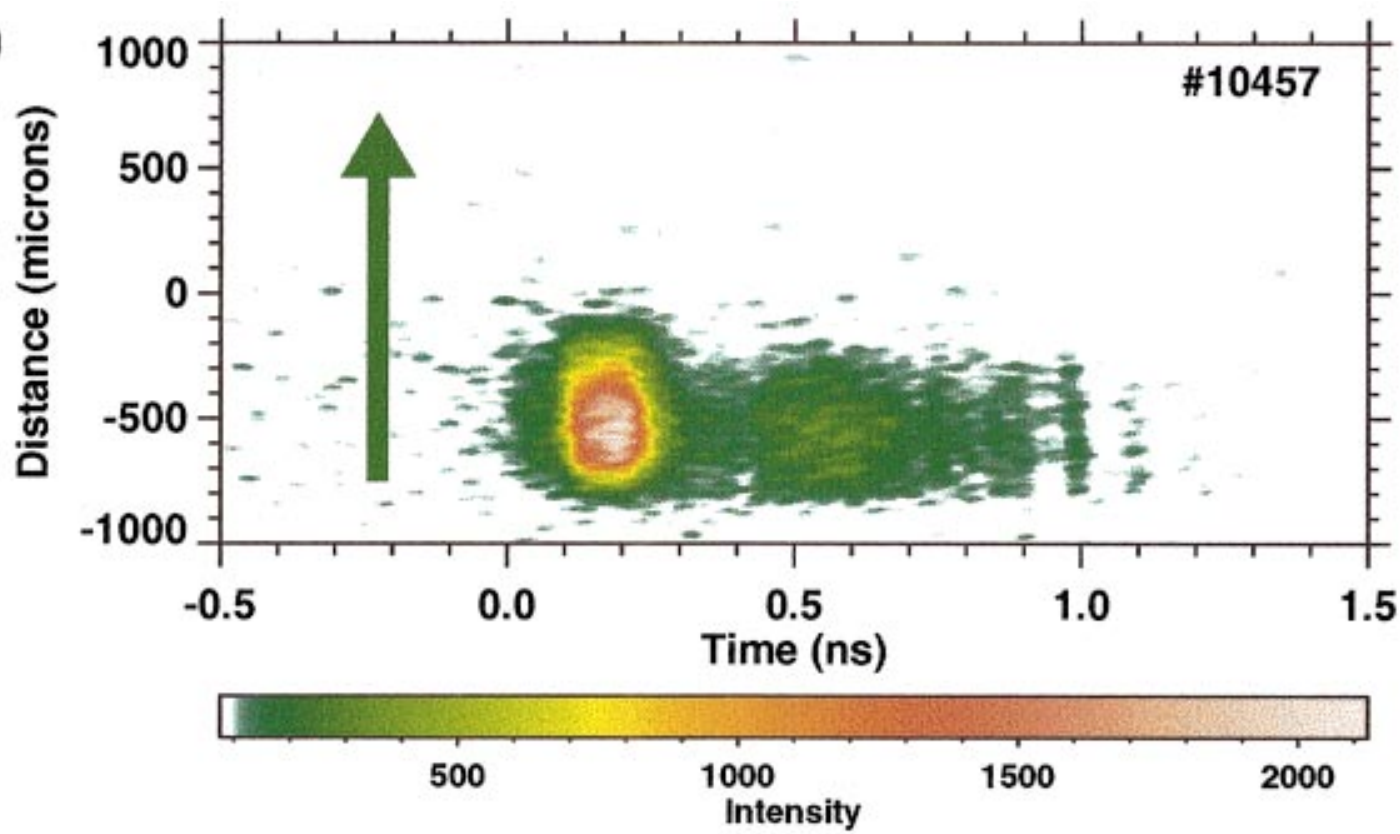

(b)

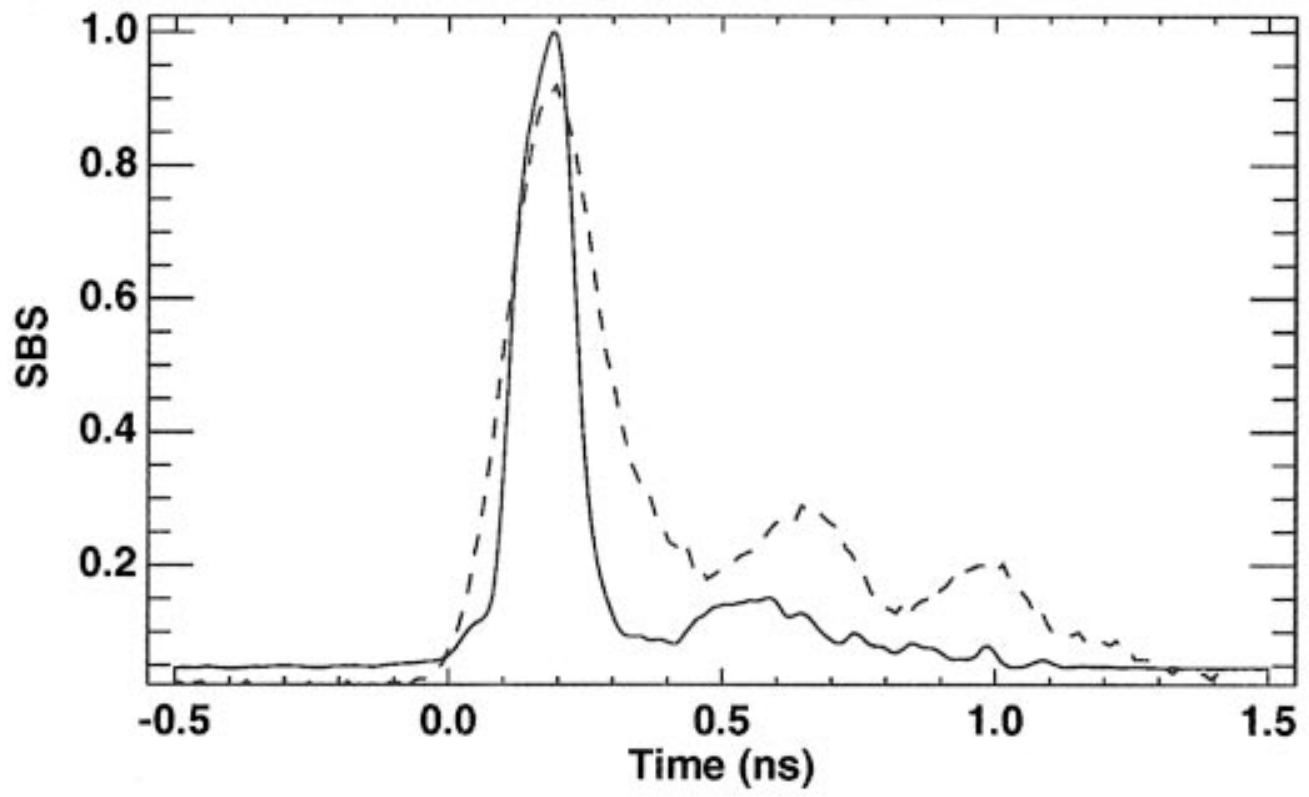

FIG. 5. (Color) Ion acoustic waves associated with SBS from the interaction beam. (a) Time-resolved position of the ion acoustic waves from Thomson scattering. The arrow is in the direction of the interaction beam. Negative values of distance denote the upstream end of the interaction beam. The target extends from -500 to $500 \mu \mathrm{m}$. (b) Time history of the ion acoustic waves from Thomson scattering (solid line) and the backscattered SBS signal of Fig. 3 (dashed line).

be stray light, which would contribute to some of the broadening of the Stokes component. However, the broadening could also be due to an angular distribution of driven ion acoustic activity. (See the discussion on NBI data below.)

\section{INVESTIGATIONS}

\section{A. Scattering from ion acoustic waves}

Details of the beam behavior and interaction with the plasma must be considered. The $150 \mu \mathrm{m}$ diameter interaction beam has a depth of focus of $\sim 750 \mu \mathrm{m}$-nearly the same as the length of the plasma. However, the speckles or hot spots which exist in the beam are shorter $-8 f^{2} \lambda$ or $\sim 150 \mu \mathrm{m} .^{23,24}$
The result is that the ion acoustic activity is not created in the plasma by a uniform homogenous beam of light (the interaction beam) but is an integration of activity from multiple speckles or hot spots in the interaction beam. How this integration occurs depends on hot spot statistics. As noted by Fernández et al., ${ }^{25}$ we expect the saturated SBS levels potentially to be sensitive to the seed perturbation for convective amplification.

In addition, as seen before, ${ }^{6,7,25,26}$ the interaction beam is deflected by refraction from the plasma density gradient and by the plasma flow as it propagates through the plasma. In the present plasma, given a uniform transverse flow and thus a constant beam deflection rate per unit length, a quadratic 

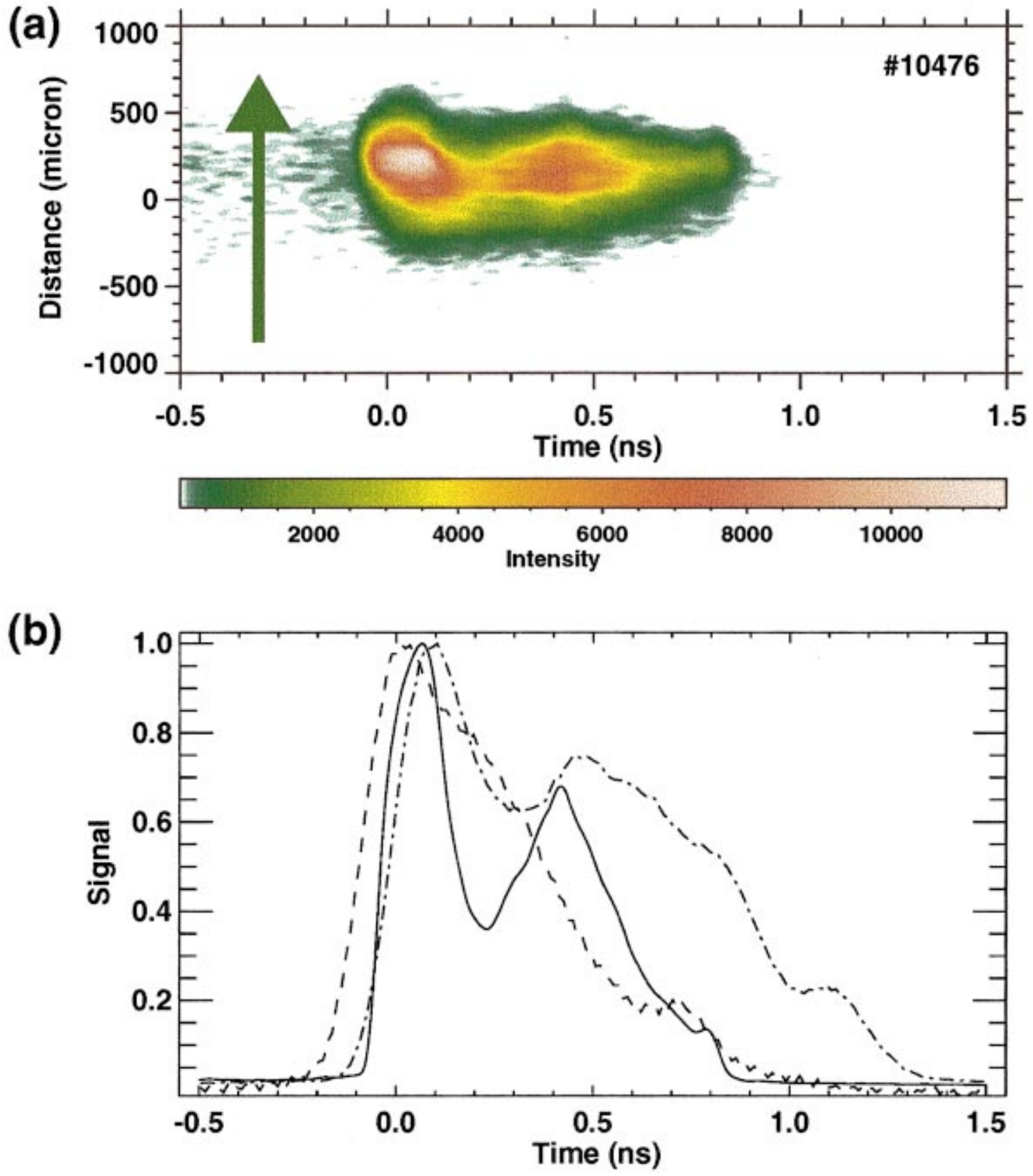

FIG. 6. (Color) Electron plasma waves associated with SRS from the interaction beam. (a) Time-resolved position of the electron plasma waves from Thomson scattering. (b) Time history of the electron plasma waves from Thomson scattering (solid line), the backscattered SRS signal (dashes), and backscattered SBS (dashes and dots). Notice the difference between the backscattered SBS here and in Fig. 5.

trajectory away from the target is expected with an overall deflection of the beam of $\sim 10^{\circ}$. The Thomson probe will be deflected also, but since it (i) is not aligned with the ribbonlike $1 \mathrm{~mm}$ length of the plasma but crosses through its $<200$ $\mu \mathrm{m}$ width and (ii) has a shorter wavelength, it will be deflected less than the interaction beam. The overlapping Thomson probe is adjusted in width to account for the interaction beam deflection to ensure that the probe does not miss the deflected interaction beam.

The imaging streak camera is appropriately filtered to transmit $351 \mathrm{~nm}$ Thomson scattered light and eliminate scattered $527 \mathrm{~nm}$ light originating from the other beams. Figure 5 shows a Thomson scattered image of the ion acoustic ac- tivity in the $1 \mathrm{~mm}$ long plasma. For all laser shots, the signal is concentrated on the upstream end of the interaction beam (where it enters the plasma). There is clearly a region about $300 \mu \mathrm{m}$ in length where the spatial gain is not exponential, but where the ion acoustic activity is approximately constant. This is suggestive of saturation. For reference, the exponentiation distance for SBS for these plasma conditions at 1.2 $\times 10^{15} \mathrm{~W} / \mathrm{cm}^{2}$ is $10-15 \mu \mathrm{m}$ and even less for hot spots of higher than average intensity. We note a shot-to-shot movement of the peak of the activity $( \pm 120 \mu \mathrm{m})$, only some of which can be attributed to systematic diagnostic alignment errors $( \pm 80 \mu \mathrm{m})$. In some instances, the signal persists beyond the nominal upstream end of the plasma, which sug- 
gests that plasma edge effects are playing a role. In order to measure a large signal, the point chosen for the Thomson spectra is $300 \mu \mathrm{m}$ upstream from the target center, near where the ion acoustic activity is strongest.

We assessed stray light by looking at the Thomson spectra and at shots without the interaction beam. The spectra are dominated by the red-shifted ion feature of the Thomson profile, which indicates growth of the ion acoustic wave. This suggests that stray $351 \mathrm{~nm}$ light is not a factor in the imaging data. Notably, in the absence of the interaction beam, when the ion acoustic activity is not pumped, the imaging streak camera detects no signal at all. Finally, the time dependence of the imaged ion acoustic activity is approximately synchronous with the SBS backscatter signal from $527 \mathrm{~nm}$ light, which indicates that the ion acoustic activity we are probing is associated with SBS. This is shown in Fig. 5(b). Stray light would not show this time history. For these reasons, we conclude that stray light is not a factor in these measurements. Thus, while the temporal behavior of the SBS is variable from shot to shot, the location is not; it is always on the upstream end of the interaction beam.

\section{B. Scattering from electron plasma waves}

Having looked at the location of ion acoustic activity associated with SBS induced by the interaction beam, we changed the filtration of the imaging streak camera to examine the location of electron plasma waves driven by the same interaction beam. At the same time, the streaked Thomson spectrometer was switched to a lower dispersion grating for determining the spectrum associated with electron plasma waves. The scattering vector diagram is slightly different [Fig. 1(c)] since $\mathbf{k}_{e p}<\mathbf{k}_{i a}$ and because of the red shift of Thomson scattered light. However, as mentioned before, the collection lens solid angle is large enough to detect light from the Thomson probe corresponding to forwardpropagating electron plasma waves. For the imaged electron plasma wave data, a $410 \mathrm{~nm}$ bandpass filter was used. The data indicate a variability of the location of the most intense electron plasma waves in the interaction beam from shot to shot. Although not active everywhere at once, electron plasma waves can exist over the entire $1 \mathrm{~mm}$ length of the plasma. Figure 6(a) shows an example of electron plasma wave scattering from a more central portion of the plasma as opposed to the usual upstream location of ion acoustic activity. Besides the electron plasma wave time history, Fig. 6(b) displays the time behavior of SBS and SRS backscatter for this shot. The lack of the expected temporal anticorrelation ${ }^{9}$ between SBS and SRS seen in the backscatter diagnostic may be due to the spatial origins of the signals. If the waves are not spatially overlapping, the question of temporal competition loses its significance. Although there may be a spatial competition due to the detuning of one of the instabilities locally, ${ }^{27}$ both SBS and SRS appear to be free to grow to saturated values in a long-scale-length plasma.

From spatial lineouts of the SBS and SRS data, we learn more about the growth rates and saturation of the electrostatic waves. Typical lineouts showing the upstream location for ion acoustic activity are seen in Fig. 7(a). The random (a)

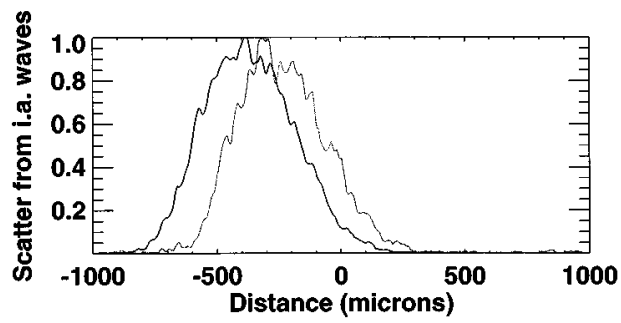

(b)

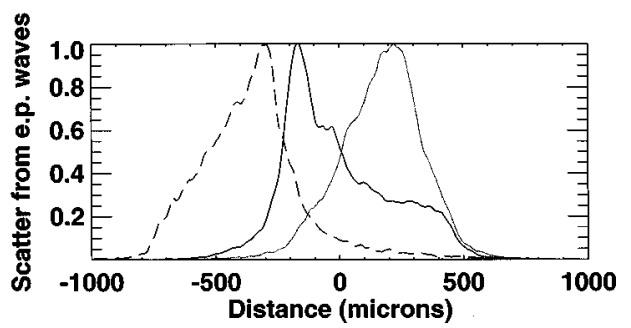

FIG. 7. Normalized lineouts from different laser shots of (a) ion acoustic and (b) electron plasma wave activity versus spatial location along the interaction beam path in the plasma.

nature of electron plasma wave activity is seen in Fig. 7(b), which graphically shows the variation of location for these waves in the plasma. For the traces in these figures, the measured e-folding length of SBS growth is $\sim 100 \mu \mathrm{m}$ while for SRS it can be as short as $60-80 \mu \mathrm{m}$. These are the average values integrated over the hot spot distribution for each transverse position of the beams (and time averaged over $0.25 \mathrm{nsec}$ ) and thus represent minimum growth lengths. Gain from single hot spots is expected to have shorter scale lengths. The width of the peak SRS activity is shorter than for SBS imaging, about the length of or shorter than a speckle length $(\sim 150 \mu \mathrm{m})$. Saturation of the SRS appears to occur within a couple of speckle lengths; however, it may turn on again further along the backscatter direction as seen in the dark solid trace of Fig. 7(b). Moreover, as seen in this same trace, the electron plasma wave apparently may also be quenched in less than a hot spot length.

The low-dispersion Thomson spectrum is shown in Fig. 8. We note that the strongest signal, exceeding the scatter from the ion acoustic wave, is located near $410 \mathrm{~nm}$. This is interpreted as scatter from interaction-beam-driven electron plasma waves. When the interaction beam is turned off, this element of the spectrum vanishes. Using the side-scattered Thomson wavelength with a correction for a $500 \mathrm{eV}$ electron temperature, we calculate the plasma density to be in agreement with the estimate obtained from SRS backscatter from the interaction beam.

An interesting phenomenological feature in the low dispersion spectrum is a short burst at $362 \mathrm{~nm}$. This is reminiscent of an observation by Labaune et $a .^{28}$ in which a red shift from $351 \mathrm{~nm}$ is proportional to the interaction beam intensity. In the present experiment, the interaction beam intensity is an order of magnitude higher than in the French experiment, and the red shift is approximately an order of magnitude greater too. The scatter was associated with ion acoustic activity by the authors. 


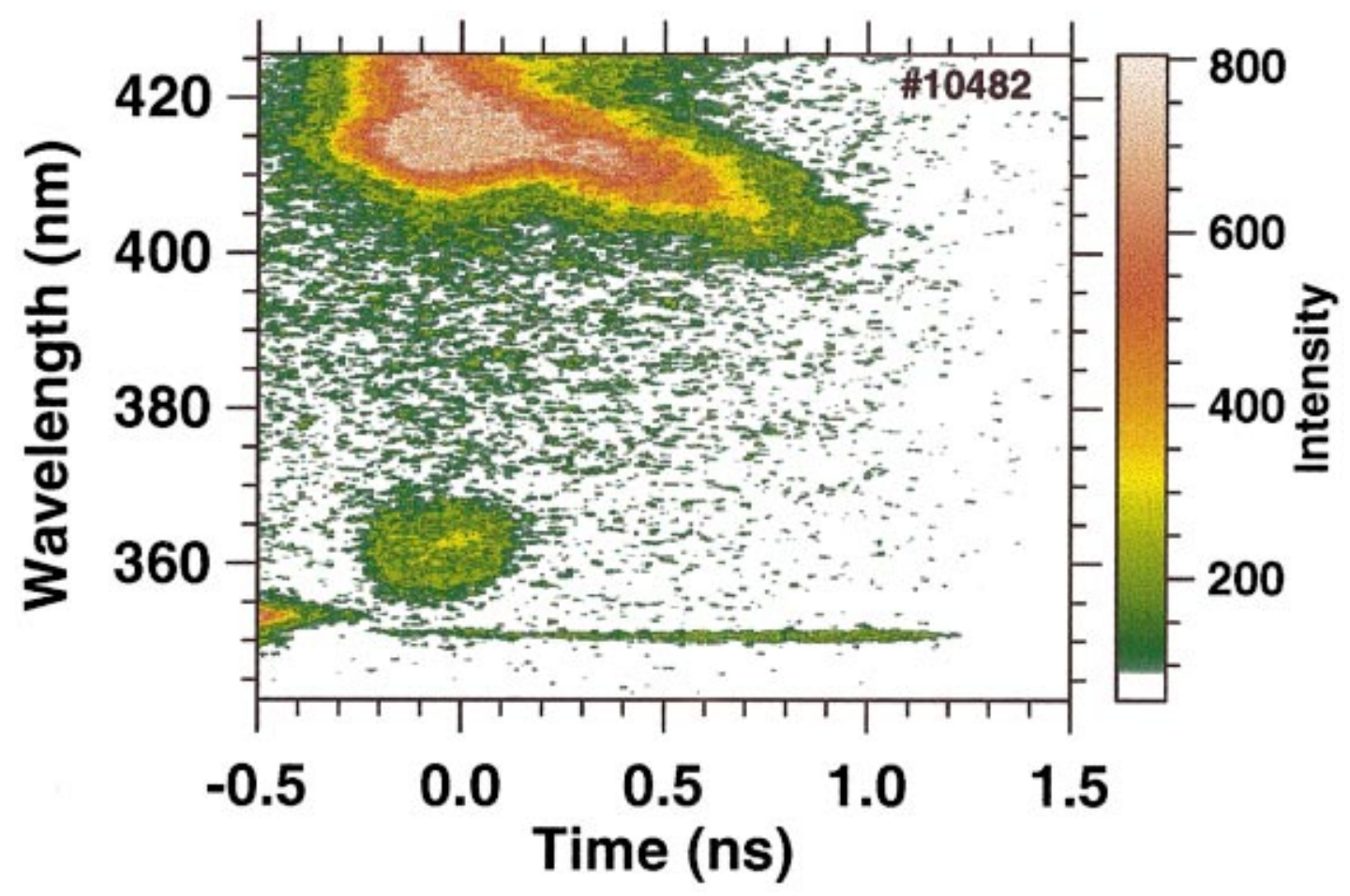

FIG. 8. (Color) Time-resolved Thomson scattering spectrum of the electron plasma waves and the ion acoustic waves.

\section{Comments on backscattered light and NBI}

The NBI diagnostic shows a deflection of the backscattered SBS light similar to other experiments. ${ }^{25}$ See Fig. 9(a). Using the $f / 6$ hole in the diffuser as a calibration, the angular deflection of the SBS from the axis is $\sim 10^{\circ}$. Thus, $\mathbf{k}_{i a}$ is not exactly parallel to the interaction beam as assumed, but deviates from it by $\sim 5^{\circ}$ (unless $\mathbf{k}_{0}$ is deflected $10^{\circ}$ in a single hot spot). Thomson scattering from this deflected ion acoustic wave is within the collection solid angle of the $f / 4$ lens. This raises an interesting question: if the primary source for the greatest SBS is ion acoustic activity on the upstream side of the plasma, before the interaction beam as a whole is significantly deflected, why is the backscattered NBI light deflected? One possible answer is that the activity is seeded by downstream SBS from regions beyond where major deflection has occurred, even if this angle is not the angle for maximum gain within the upstream hot spots. ${ }^{29} \mathrm{~A}$ second explanation, alluded to earlier, is that a single hot spot can indeed be deflected $\sim 10^{\circ}$ within its length by the combination of the transverse density gradient and transonic plasma flow. ${ }^{30}$ The dominant effect is the intensity-dependent deflection due to the flow, which is not reversible for the backscattered light on account of its lower intensity. We note that the NBI lacks the resolution to distinguish between these possibilities.

The SBS signal into the lens, as measured by the vacuum photodiode, is in the range $1.6 \% \pm 0.5 \%$ of the incident laser light energy. The center of the backscattered light however is $\sim 10^{\circ}$ toward the plane of the target and the space-integrated SBS backscatter is in the range of 5\%-10\%. This observation underlines the importance of understanding laser plasma instabilities for their control on the NIF.
Finally, to see SRS on the NBI, we used a $600 \mathrm{~nm}$ long pass filter to block $527 \mathrm{~nm}$ backscatter. We find in Fig. 9(b) that SRS is deflected in the same direction and nearly the same amount as SBS. Like the SBS, the majority of the SRS is outside the lens cone. However, we do not have a calibration of the SRS for this diagnostic. The deflection of the SRS onto the NBI is consistent with deflection of individual speckles regardless of the location of the plasma wave activity. The assumption is that the backscattered light is lower in intensity and hence not deflected by the flow exactly antiparallel to $k_{0}$.

\section{CONCLUSIONS}

We have studied with imaging Thomson scattering the strength of ion acoustic and electron plasma wave activity, associated with SBS and SRS and induced by an interaction beam in a $1 \mathrm{~mm}$ scale-length plasma. The plasma is (except for its temperature) a reasonable analog of those expected in NIF hohlraums, i.e., one having $\mathrm{a} \geqslant 1 \mathrm{~mm}$, flat-topped density profile with $n / n_{\text {crit }} \sim 5 \%$ and having a flow transverse to the beam propagation direction. We have shown examples of data which indicate the increase in ion acoustic wave activity on the upstream end of the interaction beam's passage through the plasma, a variable location for electron plasma waves, and a uniform deflection of near backscatter light for both SBS and SRS. There exists a significant length over which there is no exponential gain in ion acoustic wave activity, but, in fact, the amplitude is nearly constant, i.e., saturated. The peak of electron plasma wave activity may follow or precede a region of reduced activity. The temporal and spatial evolution of the instabilities is not systematically anticorrelated. In addition, with up to $10 \%$ total SBS (time 

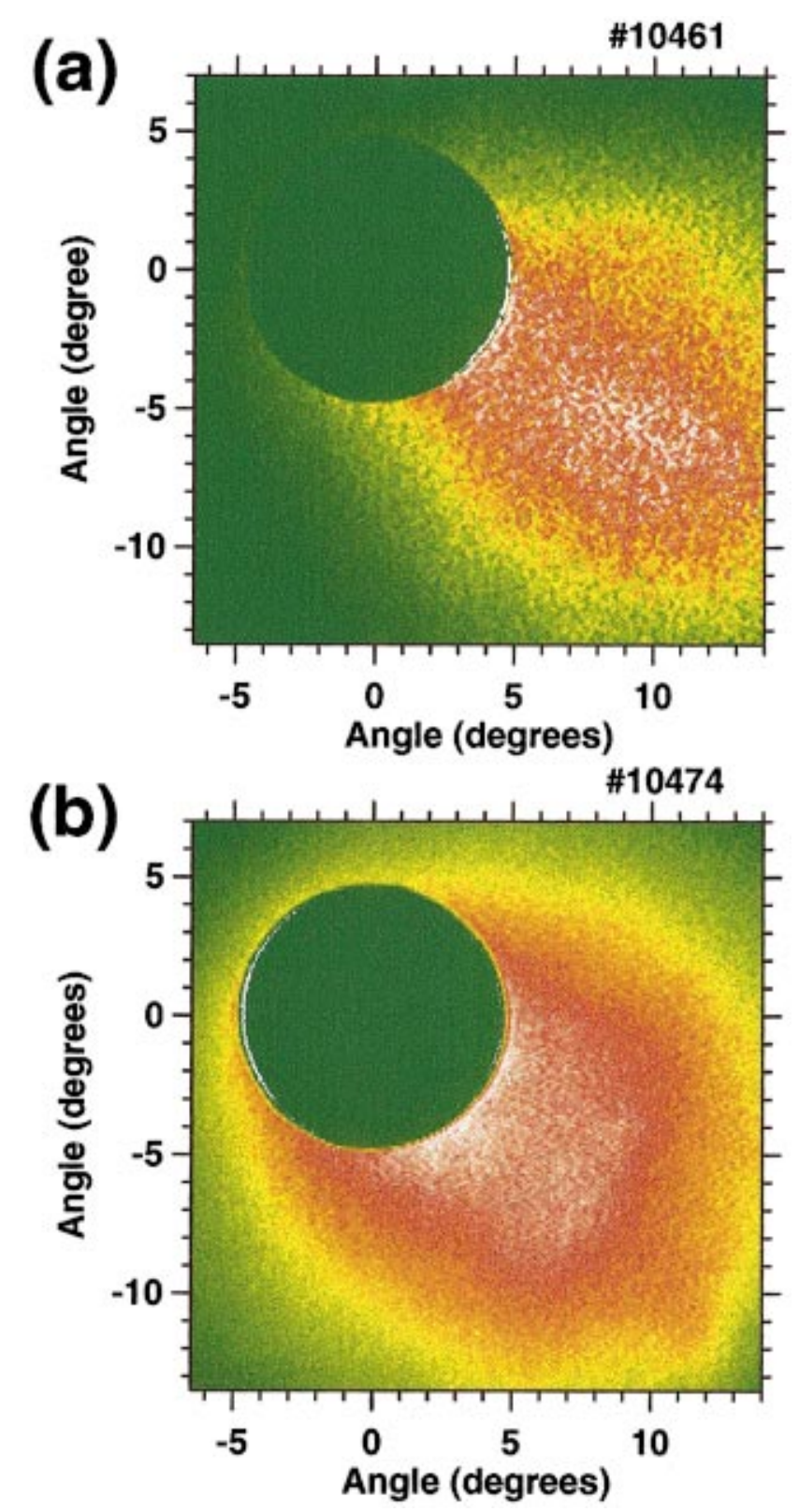

FIG. 9. (Color) The 2D images of the angular position of the backscattered light out of the lens: (a) SBS light, (b) SRS light. (The tilt is due to the camera configuration.)

integrated in the lens and on NBI), the relatively short time duration of SBS activity translates into a substantial SBS backscatter fraction for some early portions of the interaction beam.

Let us return to the root cause of the saturation of electrostatic waves in a NIF-scale plasma. The observed large spatial and temporal growth of these instabilities strongly suggests that lower-than-calculated gain rates do not account for the saturation. In fact, the rapidity with which the instabilities grow seems more than adequate to make the wave amplitudes much larger than measured were they not limited by some other mechanism. Competition between SBS and SRS seems not to be a factor in limiting either one given the modest plasma scale lengths necessary to reach relatively high values of reflectivity. Competition between modes may at some level be a saturation mechanism (perhaps only within single hot spots), but, for now, we seem not to have reached that level nor is it known what amount of reflectivity that would be. The conclusion of this work then is that other effects are moderating the saturation of SBS and SRSperhaps turbulence or hydrodynamic fluctuations.

This physics is likely to be much more complex than present detailed models can simulate for a plasma of this size. Given the present understanding of the physics of laserplasma instabilities and the potential impact these may have on the NIF, further work is warranted for developing better modeling tools and experiments to provide researchers with the means to address them on the NIF.

\section{ACKNOWLEDGMENTS}

The authors thank the following through whose faithful efforts this work was accomplished. Members of the Trident laser crew handling laser operation and target diagnostics were Nathan Okamoto, Tom Hurry, Tom Ortiz, Fred Archuleta, and Ray Gonzales. Able target fabrication support came from Joyce Moore and Veronica Gomez. John Jimerson took responsibility for fielding one of the streak cameras. Bob Harrison assisted in the construction of the Thomson beam lines. We especially thank Harvey Rose, who calculated for us single-hot-spot deflection for our flow conditions. This work was done under the auspices of the United States Department of Energy.

${ }^{1}$ R. Sawicki, J. Bowers, R. Hackel, D. Larson, K. Manes, and J. Murray, Fusion Technol. 34, 1097 (1998).

${ }^{2}$ M. N. Rosenbluth, Phys. Rev. Lett. 29, 565 (1972).

${ }^{3}$ L. M. Goldman, J. Soures, and M. J. Lubin, Phys. Rev. Lett. 31, 1184 (1973).

${ }^{4}$ H. A. Rose, Phys. Plasmas 2, 2216 (1995).

${ }^{5}$ J. C. Fernández, J. A. Cobble, B. H. Failor, W. W. Hsing, H. A. Rose, B. H. Wilde, K. S. Bradley, P. L. Gobby, R. Kirkwood, H. N. Kornblum, D. S. Montgomery, and M. D. Wilke, Phys. Rev. E 53, 2747 (1996).

${ }^{6}$ J. D. Moody, B. J. MacGowan, D. E. Hinkel, W. L. Kruer, E. A. Williams, K. Estabrook, R. L. Berger, R. K. Kirkwood, D. S. Montgomery, and T. D. Shepard, Phys. Rev. Lett. 77, 1294 (1996).

${ }^{7}$ H. A. Rose, Phys. Plasmas 3, 1709 (1996).

${ }^{8}$ D. S. Montgomery, B. B. Afeyan, J. A. Cobble, J. C. Fernández, M. D. Wilke, S. H. Glenzer, R. K. Kirkwood, B. J. MacGowan, J. D. Moody, E. L. Lindman, D. H. Munro, B. H. Wilde, H. A. Rose, D. F. Dubois, B. Bezzerides, and H. X. Vu, Phys. Plasmas 5, 1973 (1998).

${ }^{9}$ C. J. Walsh, D. M. Villeneuve, and H. A. Baldis, Phys. Rev. Lett. 53, 1445 (1984).

${ }^{10}$ J. D. Lindl, Inertial Confinement Fusion (American Institute of Physics, Springer-Verlag, New York, 1998), p. 141.

${ }^{11}$ N. K. Moncur, R. P. Johnson, R. G. Watt, and R. B. Gibson, Appl. Opt. 34, 4274 (1995).

${ }^{12}$ Y. Kato, K. Mima, N. Miyanaga, S. Arinaga, Y. Kitagawa, M. Nakatsuka, and C. Yamanaka, Phys. Rev. Lett. 53, 1057 (1984).

${ }^{13}$ S. N. Dixit, I. M. Thomas, B. W. Woods, A. J. Morgan, M. A. Henesian, P. J. Wegner, and H. T. Powell, Appl. Opt. 32, 2543 (1993).

${ }^{14}$ R. G. Watt, J. Cobble, D. F. DuBois, J. C. Fernández, H. A. Rose, R. P. Drake, and B. S. Bauer, Phys. Plasmas 3, 1091 (1996).

${ }^{15}$ D. S. Montgomery, R. P. Johnson, J. A. Cobble, J. C. Fernández, E. L. Lindman, and H. A. Rose, Laser Part. Beams 17, 349 (1999).

${ }^{16}$ A. W. DeSilva and G. C. Goldenbaum, "Plasma Diagnostics by Light Scattering," in Methods of Experimental Physics, 9A edited by H. R. Griem and R. H. Lovberg (Academic, New York, 1970), p. 61.

${ }^{17}$ H. A. Baldis, J. C. Samson, and P. B. Corkum, Phys. Rev. Lett. 41, 1719 (1978). 
${ }^{18}$ C. Labaune, H. A. Baldis, E. Schifano, B. S. Bauer, A. Michard, N. Renard, W. Seka, J. D. Moody, and K. G. Estabrook, Phys. Rev. Lett. 76, 3727 (1996)

${ }^{19}$ N. Renard, C. Labaune, H. A. Baldis, B. S. Bauer, B. Quesnel, E. Schifano, A. Michard, W. Seka, and K. G. Estabrook, Phys. Rev. Lett. 77, 3807 (1996).

${ }^{20}$ S. H. Glenzer, C. A. Back, L. J. Suter, M. A. Blain, O. L. Langden, J. D. Lindl, B. J. MacGowan, G. F. Stone, R. E. Turner, and B. H. Wilde, Phys. Rev. Lett. 79, 1277 (1997).

${ }^{21}$ R. K. Kirkwood, C. A. Back, M. A. Blain, D. E. Desenne, A. G. Dulieu, S. H. Glenzer, B. J. MacGowan, D. S. Montgomery, and J. D. Moody, Rev. Sci. Instrum. 68, 636 (1997).

${ }^{22}$ W. L. Kruer, The Physics of Laser Plasma Interactions (Addison-Wesley, Reading, MA, 1988), p. 77.

${ }^{23}$ H. A. Rose and D. F. DuBois, Phys. Fluids B 5, 590 (1993).

${ }^{24}$ H. A. Rose and D. F. DuBois, Phys. Rev. Lett. 72, 2883 (1994).
${ }^{25}$ J. C. Fernández, B. S. Bauer, K. S. Bradley, J. A. Cobble, D. S. Montgomery, R. G. Watt, B. Bezzerides, K. G. Estabrook, R. Focia, S. R. Goldman, D. B. Harris, E. L. Lindman, H. A. Rose, J. Wallace, and B. H. Wilde, Phys. Rev. Lett. 81, 2252 (1998).

${ }^{26}$ P. E. Young, C. H. Still, D. E. Hinkel, W. L. Kruer, E. A. Williams, R. L. Berger, and K. G. Estabrook, Phys. Rev. Lett. 81, 1425 (1998).

${ }^{27}$ C. Labaune, H. A. Baldis, N. Renard, E. Schifano, and A. Michard, Phys. Plasmas 4, 423 (1997).

${ }^{28}$ C. Labaune, H. A. Baldis, N. Renard, E. Schifano, S. D. Baton, A. Michard, W. Seka, R. E. Bahr, B. S. Bauer, K. Baker, and K. Estabrook, Phys. Rev. Lett. 75, 248 (1995).

${ }^{29}$ D. E. Hinkel, R. L. Berger, E. A. Williams, A. B. Langdon, C. H. Still, and B. F. Lasinski, Phys. Plasmas 6, 571 (1999).

${ }^{30}$ H. A. Rose, Los Alamos National Laboratory, private communication (1999). 